\title{
Research Article \\ Variation of the Equator due to a Highly Inclined and Eccentric Disturber
}

\section{Clair do Nascimento and Tadashi Yokoyama}

Universidade Estadual Paulista, IGCE-DEMAC Caixa Postal 178, CEP 13.500-970 Rio Claro, SP, Brazil

Correspondence should be addressed to Tadashi Yokoyama, tadashi@ms.rc.unesp.br

Received 18 June 2009; Accepted 19 October 2009

Recommended by Dane Quinn

The New Horizons project is currently in progress, but until recently years ago, a mission to Neptune-Triton System predicted in the mid term 2008-2013 was one of the priorities of NASA's Solar System Exploration theme. Any way, it is important to increase our knowledge on the dynamics of the inner satellites of Neptune, since according to some authors, this was a key question in the mission to Neptune-Triton system. In a previous work, we presented the expansion of the disturbing function for the dynamics of this system. Here we derive the averaged classical equations of the precession of the equator for this problem. The highly inclined and retrograde orbit of Triton makes this problem very unusual. Therefore, the classical truncations in the inclinations are not acceptable, so that the precession equations must be obtained in closed form for the inclination. With a significant mass and due to its distance from Neptune, which is continuously decreasing, Triton should exert in the future, important precession on Neptune's equator. The effects of this precession on the inner satellites are shown, including some resonant cases predicted in the future. Although Triton's orbit is almost circular, no expansion in the eccentricity is needed.

Copyright (C) 2009 C. do Nascimento and T. Yokoyama. This is an open access article distributed under the Creative Commons Attribution License, which permits unrestricted use, distribution, and reproduction in any medium, provided the original work is properly cited.

\section{Introduction}

Some years ago, the exploration of the Neptune system in the next ten years was posed as an important priority of NASA's Solar System Exploration theme [1]. Although the New Horizons project is now the top priority for the far reaches of the solar system, most probably, to design a mission to explore the Neptune-Triton system will be just a mater of time. The authors [1] raised some important questions about the dynamics (stability) of the arcs and inner satellites of Neptune. In order to give support to the mission, we must increase our knowledge on the dynamics of this system. In this work we derive the classical equations of the precession for the equator of Neptune. Basically, this equator is disturbed by the presence of Triton since it has a remarkable mass: $m_{T} / M_{N} \approx 2.09 \times 10^{-4} \cdot\left(m_{T}, M_{N}\right.$ are 


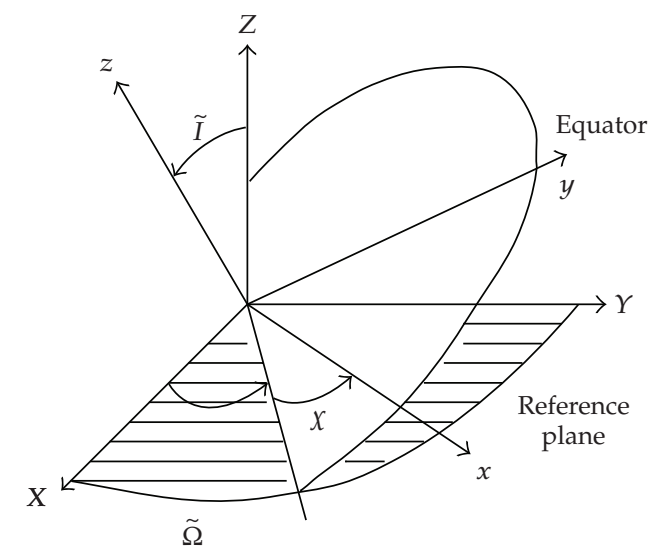

Figure 1: XYZ: inertial system, $x y z$ : moving system fixed on the equator.

the masses of Triton and Neptune, resp.). Moreover, Triton's inclination with respect to Neptune's equator is quite unusual $\left(\approx 157.35^{0}\right)$. In a short time span, this precession should not be so important on the dynamics of the inner satellites. However, for a longer time (more than 600 years), this effect must be investigated. This work is organized in the following way: in Section 2 the averaged precessional equations for Neptune's equator considering arbitrary inclination obtained. Some authors [2] hypothesize that in the past, the eccentricity of Triton $\left(e_{T}\right)$, might have been very high. Therefore, the precessional equations are also obtained for arbitrary $e_{T}$. In Section 3, we discuss the definition of the invariable plane and the existence of a corresponding equilibrium solution when we assume circular orbit for Triton. Considering the current dynamics of Neptune-Triton system, several authors have improved the ephemerides and data of the small satellites (e.g., Owen et al. [3]). Here, some numerical simulations showing the effects of the precession, particularly for the future $2: 1$ and $3: 1$ resonances involving Triton and Proteus, are presented. Section 4 is devoted to the conclusions.

\section{The $\tilde{I}$ and $\widetilde{\Omega}$ equations}

Let $X Y Z$ be an arbitrary reference system (inertial) and a moving system $x y z$ fixed on the equator of Neptune (see Figure 1). We define $\widetilde{I}=$ inclination of the equator and $\widetilde{\Omega}=$ node of the equator [4]. Due to the presence of a massive satellite (Triton), the equator of Neptune will be disturbed. Let $\mathrm{T}$ be the associated torque due to Triton and let $W_{T}$ be the corresponding oblateness potential of Neptune:

$$
W_{T}=-\frac{G M_{N} m_{T} J_{2} R_{N}^{2}}{r_{T}^{3}}\left(-\frac{1}{2}+\frac{3 \sin ^{2} \beta}{2}\right),
$$

where $G$ is the gravitation constant, $M_{N}$ and $m_{T}$ are the masses of Neptune and Triton, respectively. The oblateness constant is $J_{2}, \beta$ is the Triton's latitude and $r_{T}$ is the radial distance 
Neptune-Triton in the $x y z$ system. The equatorial radius of Neptune is indicated by $R_{N}$. Then:

$$
\mathbf{T}=(L, M, N)=-\mathbf{r}_{\mathbf{T}} \times \nabla_{T} W_{T},
$$

where $\nabla_{T}$ is the usual gradient operator with respect to Triton coordinates and $L, M$, and $N$ are the components of the torque $T$, that is:

$$
\begin{gathered}
L=\left(\frac{\partial W_{T}}{\partial y_{T}}\right) z_{T}-\left(\frac{\partial W_{T}}{\partial z_{T}}\right) y_{T} \\
M=-\left(\frac{\partial W_{T}}{\partial x_{T}}\right) z_{T}+\left(\frac{\partial W_{T}}{\partial z_{T}}\right) x_{T} \\
N=\left(\frac{\partial W_{T}}{\partial x_{T}}\right) y_{T}-\left(\frac{\partial W_{T}}{\partial y_{T}}\right) x_{T}
\end{gathered}
$$

where $x_{T}, y_{T}$ and $z_{T}$ are the cartesian coordinates of Triton with respect to the moving system $x y z$. On account of the rotational symmetry, we can show that $N=0$. To this end, since $\beta$ is the latitude we have: $\sin (\beta)=z_{T} / r_{T}$. Then a direct calculation shows that $W_{T}$ depends on Triton coordinates only through $r_{T}$ and $z_{T} / r_{T}$, therefore:

$$
\begin{gathered}
W_{T}=W_{T}\left(r_{T}, \frac{z_{T}}{r_{T}}\right), \quad r_{T}=\left(x_{T}^{2}+y_{T}^{2}+z_{T}^{2}\right)^{1 / 2}, \\
\frac{\partial W_{T}}{\partial x_{T}}=\frac{\partial W_{T}}{\partial r_{T}} \frac{x_{T}}{r_{T}}, \quad \frac{\partial W_{T}}{\partial y_{T}}=\frac{\partial W_{T}}{\partial r_{T}} \frac{y_{T}}{r_{T}} .
\end{gathered}
$$

Substituting these last two relations in $N$ given in (2.3), we get $N=0$. Now, let $\mathbf{w}=$ $\left(p_{N}, q_{N}, w_{N}\right)$ be the rotation vector of Neptune. According to Euler's dynamical equations:

$$
\begin{gathered}
\dot{p}_{N}+\frac{C-A}{A} w_{N} q_{N}=\frac{L}{A}, \\
\dot{q}_{N}-\frac{C-A}{A} w_{N} p_{N}=\frac{M}{A}, \\
\dot{w}_{N}=\frac{N}{C}=0,
\end{gathered}
$$

where $A(=B)$ and $C$ are the moments of inertia of the planet. From the geometric equations, $\mathbf{w}$ can be expressed in terms of the inertial XYZ system:

$$
\begin{gathered}
p_{N}=\sin (\widetilde{I}) \sin (x) \dot{\widetilde{\Omega}}+\cos (X) \dot{\tilde{I}}, \\
q_{N}=\sin (\widetilde{I}) \cos (X) \dot{\widetilde{\Omega}}-\sin (x) \dot{\tilde{I}}, \\
w_{N}=\cos (\widetilde{I}) \dot{\widetilde{\Omega}}+\dot{X} .
\end{gathered}
$$


Taking the time derivative of $p_{N}$,

$$
\begin{aligned}
\dot{p}_{N}= & \cos (\widetilde{I}) \dot{\tilde{I}}(\sin (x) \dot{\widetilde{\Omega}})+\sin (\widetilde{I})(\dot{X} \cos (x) \dot{\widetilde{\Omega}}+\sin (x) \ddot{\tilde{\Omega}}) \\
& -\sin (\chi) \dot{X} \dot{\widetilde{I}}+\cos (\chi) \ddot{\widetilde{I}}
\end{aligned}
$$

Assuming that the polar component of the rotation of the planet is dominant, the following quantities: $\dot{\widetilde{I}} \dot{\widetilde{\Omega}}, \ddot{\widetilde{I}}$ e $\ddot{\widetilde{\Omega}}$ e $\dot{\widetilde{\Omega}}^{2}$ can be neglected when compared to $\dot{X}[5,6]$. Then,

$$
\dot{p}_{N}=\sin (\widetilde{I}) \dot{X} \cos (x) \dot{\widetilde{\Omega}}-\sin (x) \dot{X} \dot{\tilde{I}}
$$

Substituting $\dot{p}_{N}$ e $q_{N}$ in the first Euler's equation,

$$
\begin{aligned}
\frac{L}{A}= & (\dot{\widetilde{\Omega}} \dot{\chi} \cos (\chi) \sin (\widetilde{I})-\dot{\tilde{I}} \dot{\chi} \sin (\chi)) \\
& +\frac{C}{A} w_{N}(\dot{\widetilde{\Omega}} \sin (\widetilde{I}) \cos (\chi)-\sin (\chi) \dot{\tilde{I}}) \\
& -w_{N}(\dot{\tilde{\Omega}} \sin (\tilde{I}) \cos (\chi)-\sin (\chi) \dot{\tilde{I}})
\end{aligned}
$$

Substituting $w_{N}=\cos (\widetilde{I}) \dot{\widetilde{\Omega}}+\dot{X}$ and neglecting terms factored by $\dot{\widetilde{\Omega}}^{2}$ and $\dot{\widetilde{I}} \dot{\widetilde{\Omega}}$, we have

$$
\frac{C}{A} w_{N}(\sin (\widetilde{I}) \cos (x) \dot{\widetilde{\Omega}}-\sin (x) \dot{\tilde{I}})=\frac{L}{A}
$$

Similarly, putting $\dot{q}_{N}$ and $p_{N}$ into the second Euler dynamical equation, we obtain:

$$
-\frac{C}{A} w_{N}(\sin (\tilde{I}) \sin (\chi) \dot{\tilde{\Omega}}+\cos (\chi) \dot{\tilde{I}})=\frac{M}{A}
$$

Now we can eliminate $L$ and $M$ in the above equations. Defining $W_{0}=-G m_{T} M_{N} J_{2} R_{N}^{2} / 2$, we have

$$
\begin{gathered}
W_{T}=W_{0}\left(\frac{3 z_{T}^{2}}{r_{T}^{5}}-\frac{1}{r_{T}^{3}}\right), \\
\frac{\partial W_{T}}{\partial y_{T}}=W_{0}\left[\frac{\partial}{\partial r_{T}}\left(\frac{3 z_{T}^{2}}{r_{T}^{5}}-\frac{1}{r_{T}^{3}}\right)\right] \frac{y_{T}}{r_{T}} \\
\frac{\partial W_{T}}{\partial z_{T}}=W_{0}\left[\frac{\partial}{\partial r_{T}}\left(\frac{3 z_{T}^{2}}{r_{T}^{5}}-\frac{1}{r_{T}^{3}}\right)\right] \frac{z_{T}}{r_{T}}+W_{z}
\end{gathered}
$$


and so,

$$
L=\frac{\partial W_{T}}{\partial y_{T}} z_{T}-\frac{\partial W_{T}}{\partial z_{T}} y_{T}=-W_{z} y_{T}
$$

where $W_{z}=6 W_{0} z_{T} / r_{T}^{5}$. Similarly, $M=W_{z} x_{T}$. From (2.10) and (2.11),

$$
\begin{gathered}
\dot{\widetilde{\Omega}} \sin (\widetilde{I})=-\frac{W_{z}}{C w_{N}}\left(y_{T} \cos (x)+x_{T} \sin (x)\right), \\
\dot{\tilde{I}}=\frac{W_{z}}{C w_{N}}\left(-x_{T} \cos (x)+y_{T} \sin (x)\right) .
\end{gathered}
$$

If a sequence of three rotations, $\widetilde{\Omega}, \widetilde{I}$, is $X$ are applied on the system $X Y Z$, it will coincide with $x y z$. Therefore we can write $x_{T}, y_{T}$, and $z_{T}$ in terms of the well-known elliptic orbital elements of Triton.

$$
\begin{gathered}
x_{T}=r_{T} \cos \left(f_{T}+w_{T}\right) \cos \Omega_{T}-r_{T} \sin \left(f_{T}+w_{T}\right) \sin \Omega_{T} \cos I_{T}, \\
y_{T}=r_{T} \cos \left(f_{T}+w_{T}\right) \sin \Omega_{T}+r_{T} \sin \left(f_{T}+w_{T}\right) \cos \Omega_{T} \cos I_{T}, \\
z_{T}=r_{T} \sin \left(f_{T}+w_{T}\right) \sin I_{T},
\end{gathered}
$$

where $r_{T}, f_{T}, w_{T}, \Omega_{T}, w_{T}$, and $I_{T}$ refer to classical orbital elements of Triton referred to $(X, Y, Z)$ inertial system.

Finally, averaging the resulting equations with respect to the mean anomaly of Triton, the precessional equations, without any restriction on the inclination $I_{T}$ or on the eccentricity $e_{T}$, are

$$
\begin{gathered}
\dot{\tilde{I}}=\frac{F_{0}}{4 a_{T}^{3}\left(1-e_{T}^{2}\right)^{3 / 2}}\left[\sin (\widetilde{I}) \sin \left(I_{T}\right)^{2} \sin \left(2 \widetilde{\Omega}-2 \Omega_{T}\right)+\cos (\widetilde{I}) \sin \left(2 I_{T}\right) \sin \left(\widetilde{\Omega}-\Omega_{T}\right)\right] \\
\dot{\widetilde{\Omega}}=\frac{F_{0}}{4 a_{T}^{3}\left(1-e_{T}^{2}\right)^{3 / 2}}\left[\left(1-3 \cos \left(I_{T}\right)^{2}\right) \cos (\widetilde{I})+\frac{2 \sin \left(I_{T}\right) \cos \left(I_{T}\right) \cos (2 \tilde{I}) \cos \left(\widetilde{\Omega}-\Omega_{T}\right)}{\sin (\widetilde{I})}\right. \\
\left.+\sin \left(I_{T}\right)^{2} \cos (\widetilde{I}) \cos \left(2 \widetilde{\Omega}-2 \Omega_{T}\right)\right]
\end{gathered}
$$

where

$$
F_{0}=\frac{3 G m_{T} M_{N} R_{N}^{2} J_{2}}{C w_{N}}
$$

Notice that, as expected in this approximation, these two equations are decoupled from $x$ equation. 


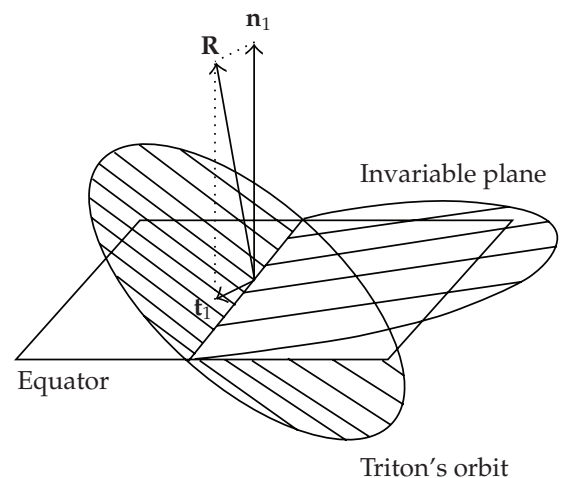

(a)

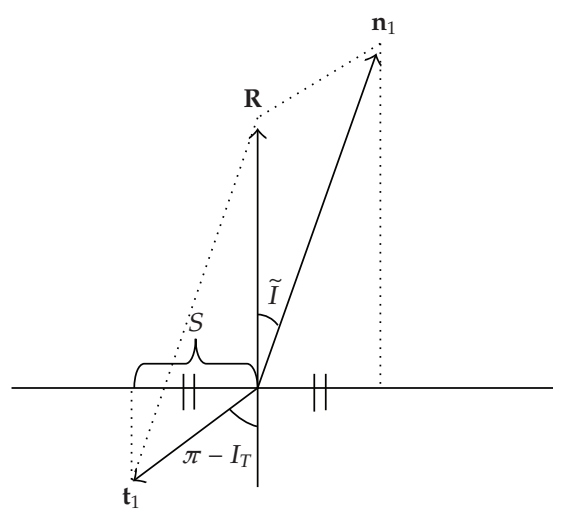

(b)

Figure 2: $\mathbf{t}_{\mathbf{1}}$ is perpendicular to the orbital plane of Triton, $\mathbf{n}_{\mathbf{1}}$ is perpendicular to Neptune's equator, and $\mathbf{R}$ is the resulting vector which defines the invariable plane.

\section{The Invariable Plane}

In this section, we define the reference plane. Let $\mathbf{t}_{1}$ and $\mathbf{n}_{\mathbf{1}}$ be the orbital angular momentum of Triton and rotation angular momentum of Netpune, respectively. Then $\mathbf{R}=\mathbf{t}_{\mathbf{1}}+\mathbf{n}_{\mathbf{1}}$ is a constant vector. Therefore, the plane perpendicular to the direction of $\mathbf{R}$ is invariable (see Figure 2), and it will be adopted as our reference plane.

The dynamics of the system involving $\widetilde{I}, \widetilde{\Omega}, \Omega_{T}$, and $I_{T}$ shows an interesting solution in this invariable plane. To see that, let us assume Triton in a circular Keplerian orbit. In this case, we have $t_{1}=m_{T} n_{T} a_{T}^{2}$, where $n_{T}$ is the mean motion, and $m_{T}$ is the Triton's mass. On the other hand, $n_{1}=C w_{N}$, where $C$ is the polar moment of inertia of Neptune and $w_{N}$ is its angular velocity of rotation (spin rate). Now, from Figure 2, we have

$$
\sin \left(\pi-I_{T}\right)=\frac{s}{m_{T} n_{T} a_{T}^{2}}, \quad \sin \tilde{I}=s C w_{N}
$$

that is, the inclination $\tilde{I}$ of the invariable plane is given by:

$$
\sin \tilde{I}=\frac{m_{T} n_{T} a_{T}^{2} \sin I_{T}}{C w_{N}}
$$

In order to have the complete set of equations that govern the motion of the equator, we have to consider (2.16) and also the corresponding equations for $I_{T}$ and $\Omega_{T}$. They are

$$
\begin{aligned}
\frac{d I_{T}}{d t} & =-\frac{1}{n_{T} a_{T}^{2} \sin \left(I_{T}\right)} \frac{\partial R_{J_{2}}}{\partial \Omega_{T}}, \\
\frac{d \Omega_{T}}{d t} & =\frac{1}{n_{T} a_{T}^{2} \sin \left(I_{T}\right)} \frac{\partial R_{J_{2}}}{\partial I_{T}},
\end{aligned}
$$


where $R_{J_{2}}$ is the disturbing function due to the oblateness of Neptune which is written with respect to the following invariable plane:

$$
\begin{aligned}
R_{J_{2}}=S_{0}[ & \frac{1}{2}\left(1-\frac{3}{2} \sin ^{2} \tilde{I}\right)\left(1-\frac{3}{2} \sin ^{2} I_{T}\right) \\
& \left.+\frac{3}{8} \sin (2 \tilde{I}) \sin \left(2 I_{T}\right) \cos \left(\Omega_{T}-\tilde{\Omega}\right)+\frac{3}{8} \sin ^{2} \tilde{I} \sin ^{2} I_{T} \cos \left(2 \Omega_{T}-2 \widetilde{\Omega}\right)\right],
\end{aligned}
$$

where

$$
S_{0}=\frac{G M_{N} R_{N}^{2} J_{2}}{\left[a_{T}^{3}\left(1-e_{T}^{2}\right)^{3 / 2}\right]}
$$

For $\widetilde{\Omega}-\Omega_{T}=\pi$, we easily see that

$$
\begin{gathered}
\dot{I}_{T}=\tilde{I}=0, \\
\dot{\widetilde{\Omega}}=-\frac{S_{2}}{\sin (\tilde{I})} \sin \left(2 \widetilde{I}+2 I_{T}\right), \\
\dot{\Omega}_{T}=-\frac{3}{4} \frac{G M_{N} R_{N}^{2} J_{2}}{n_{T} a_{T}^{5} \sin I_{T}} \sin \left(2 \tilde{I}+2 I_{T}\right),
\end{gathered}
$$

where

$$
S_{2}=\frac{3 G m_{T} M_{N} R_{N}^{2} J_{2}}{4 C w_{N} a_{T}^{3}}
$$

Therefore,

$$
\dot{\widetilde{\Omega}}-\dot{\Omega}_{T}=\frac{3 G M_{N} R_{N}^{2} J_{2}}{4 a_{T}^{3}} \sin \left(2 \tilde{I}+2 I_{T}\right)\left[\frac{1}{n_{T} a_{T}^{2} \sin I_{T}}-\frac{m_{T}}{C w_{N} \sin (\tilde{I})}\right]
$$

that is, the term inside the brackets is automatically zero provided the reference plane is the invariable plane (see (3.2)). In this case; $\dot{\widetilde{\Omega}}-\dot{\Omega}_{T}=0, I_{T}$ and $\tilde{I}$ are constants; $\widetilde{\Omega}$ and $\Omega_{T}$ are synchronous. This means that the precession rate of the Neptune's equator is the same of the Triton's node on the invariable plane $\left(\approx 688\right.$ years). It is also easy to show that $\widetilde{\Omega}-\Omega_{T}=\pi$ defines a stable periodic solution of the system (simple calculations show that such solution does not exist for $\widetilde{\Omega}-\Omega_{T}=0$ ). This means that it is very convenient to choose the invariable plane as the reference for our purposes. 


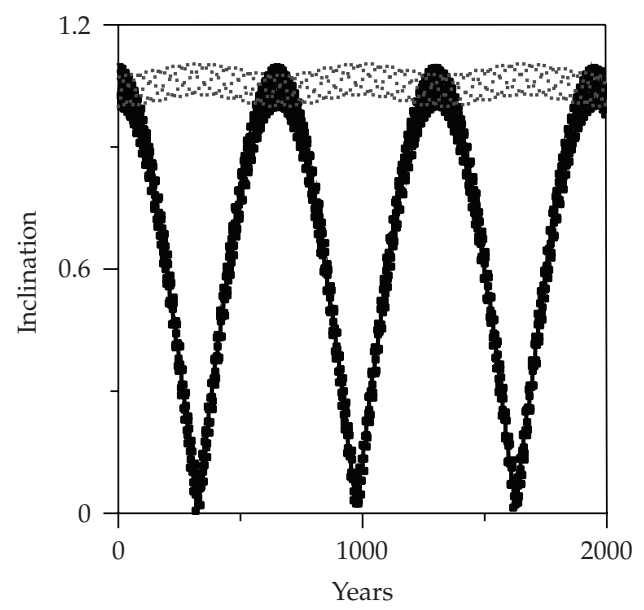

(a)

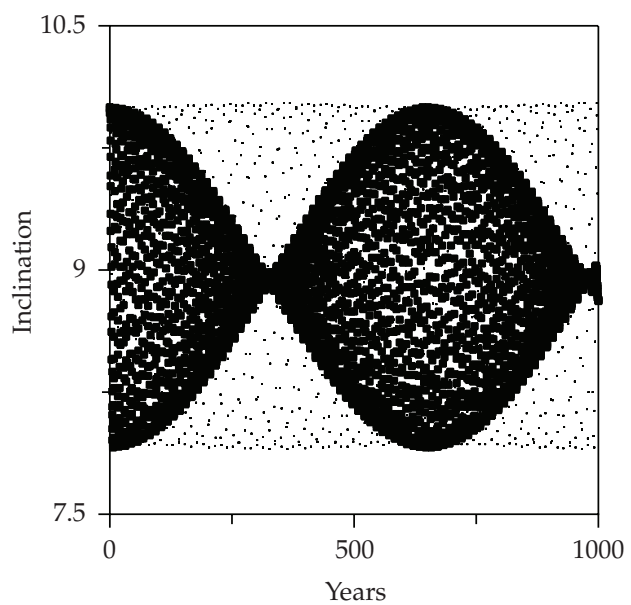

(c)

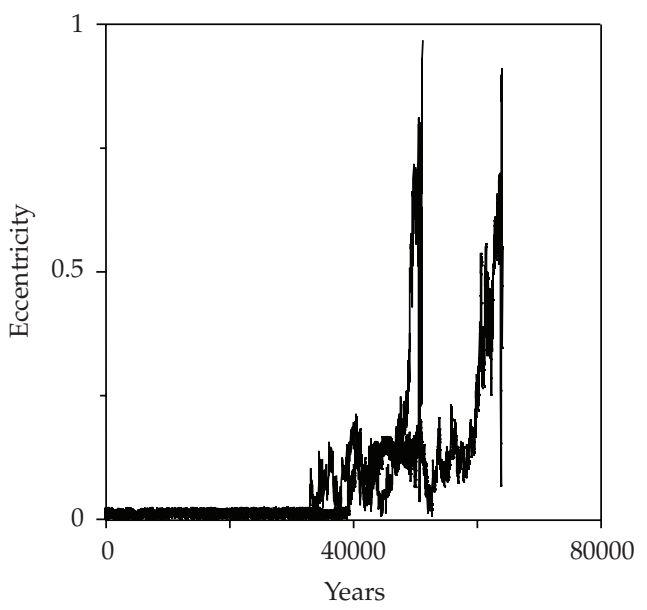

(b)

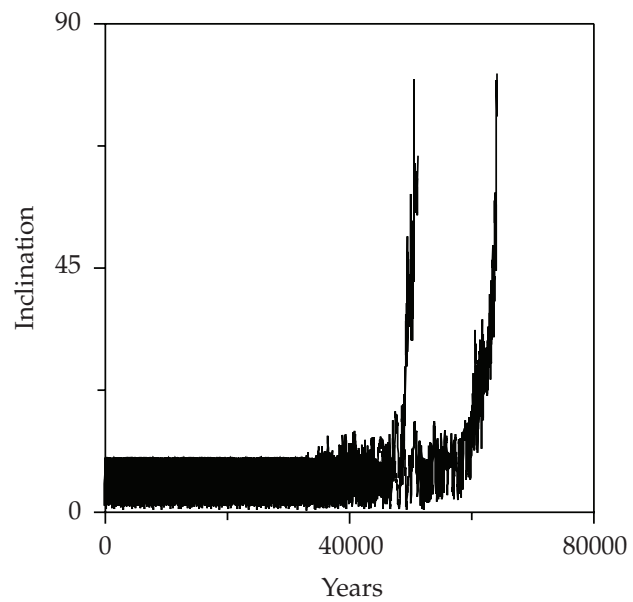

(d)

Figure 3: (a), (c): the lighter points were obtained taking into account the $\tilde{I}$ and $\dot{\widetilde{\Omega}}$ equations in the integration, while in the thick points these contributions were neglected. (b), (d): time variation of the eccentricity (b) and inclination (d) for two orbits. Initially their paths are similar, but when their semimajor axes enter the $2: 1$ resonance region, a jump appears in the eccentricity (b), first in the orbit which contains the effect of the precession. The same occurs for the inclination (d), that is, the precession accelerates the onset of the jump of the inclination.

\section{Some Effects on the Inner Satellites}

Initially we tested our (2.16) for the case of Earth and Mars. As expected, the results agree quite well with very known values $(\approx 26000$ and $\approx 175000$ years for Earth and Mars, resp.). For the Neptune's equator, considering $a_{T}=14.063 R_{N}$, our numerical experiments show $\approx 688$ years for the period of precession, referred to the invariable plane Neptune-Triton. This period is rather sensitive for the adopted value of the semimajor axis of Triton. In this section we test the effect of the precession of Neptune's equator on an inner satellite of this planet. In Figure 3, we always integrate two orbits. The lighter points in the left panels, correspond to the orbits when we include the precessional effect, while the thick points come from the orbit 
when we neglect (2.16). The inclinations are referred to the invariable plane. We consider a test particle (Proteus) disturbed by Triton.

In the integration of the equations of the motion, we used our averaged disturbing function $R$ as described Yokoyama et al. [7] which is very efficient for this case. The initial conditions for Proteus are $a=4.751, e=0.0005, w=0^{0}, \Omega=90^{\circ}, I=1^{0}$ Figure 3(a) and $I=10^{\circ}$ Figure 3(c), and $\lambda=90^{\circ}$, while for Triton we consider $a=14.325, e=0.000016, w=0^{0}$, $\Omega=270^{\circ}, I=157^{\circ}$, and $\lambda=270^{\circ}$. The effect of the precession is very clear: Figure 3(a), shows the variation of Proteus' inclination which remains confined in a very narrow interval when precession equations are considered. In Figure 3(c), in opposition to the above panel, we take Proteus at high inclination $\left(10^{\circ}\right)$. Then, although the periods are different, the inclinations of both orbits undergo a large variation. Moreover, there is quite clear a periodic oscillation of about 670 years, which is related to the precession of the node $\left(\Omega_{T}\right)$. Panels on the right, refer to the variation of the eccentricity Figure 3(b) and inclination Figure 3(d) of Proteus in the case of future 2: 1 resonance. As described by Yokoyama [8] and Yokoyama et al. [7], due to the tides, Triton is falling down to the planet, so that, its orbital period will be in a $3: 1$ or $2: 1$ orbital resonance with Proteus in the future. For these two panels, instead of the averaged equations, we integrated the exact equations and we applied a slow dissipation on the semimajor axis of Triton in order to simulate the tidal effect. As pointed in Yokoyama et al. [7] the eccentricity suffers a strong variation and Proteus can be ejected. The effect of the precession is to anticipate the ejection. However, this is not a general rule, since some tests performed in the $3: 1$ resonance showed different results, that is, the precession can delay the ejection. More tests must be carried on in order to clarify these details.

\section{Conclusion}

In this work, we derived the classical averaged equations for the precession of the Neptune's equator, extending for the case of arbitrary inclination and eccentricity of the disturber. Due to the high retrograde inclination of Triton, these equations must be obtained in closed form for this inclination. If the invariable plane is chosen as the reference plane, the averaged equations of the precession in the case of $e_{T}=0$, satisfy an equilibrium solution where $\widetilde{\Omega}$ and $\Omega_{T}$ remain synchronous while $\tilde{I}$ and $I_{T}$ are constants. The numerical experiments show that, when the precessional equations are considered, the inclination of an inner satellite of Neptune (Proteus) remains confined in a very narrow range, that is, the variation of the inclination with respect to the invariable plane is very small. However for high initial inclination $\left(\geq 10^{0}\right)$ of the inner satellite, this confinement is not preserved anymore, that is, even considering the precessional effect, the amplitude of the variation of the inclination is significant. Additional simulations show that the precessional effects are also important in the future $2: 1$ and $3: 1$ resonances, since the jump in inclination or eccentricity can be anticipated or delayed.

\section{Acknowledgments}

The authors thank FAPESP, CNPQ, and FUNDUNESP. 


\section{References}

[1] H. B. Hammel, K. H. Baines, J. D. Cuzzi, et al., "Exploration of the Netpune system," in The Future of Solar System Exploration, 2003-2013: Community Contributions to the NRC Solar System Exploration Decadal Survey, M. V. Sykes, Ed., vol. 272 of ASP Conference Series, pp. 297-323, 2002.

[2] P. Goldreich, N. Murray, P. Y. Longaretti, and D. Banfield, “Neptune's story," Science, vol. 245, pp. 500-504, 1989.

[3] W. M. Owen Jr., R. M. Vaughan, and S. P. Synnott, "Orbits of the six new satellites of Neptune," Astronomical Journal, vol. 101, no. 4, pp. 1511-1515, 1991.

[4] E. K. Woolard, Astronomical Papers of American Ephemeris, vol. 15, part 1, 1953.

[5] R. Kurth, Introduction to the Mechanics of the Solar System, Pergamon Press, London, UK, 1959.

[6] D. Lazzaro, "Semi-Analytical Theory for the Motion of Neptune's Satellites," Doctor thesis, Universidade de São Paulo, São Paulo, Brazil, 1987.

[7] T. Yokoyama, C. Nascimento, and M. T. Santos, "Inner satellites of Neptune-I: the mutual disturbing function," Advances in Space Research, vol. 36, pp. 569-577, 2005.

[8] T. Yokoyama, "Possible effects of secular resonances in Phobos and Triton," Planetary and Space Science, vol. 50, no. 1, pp. 63-77, 2002. 


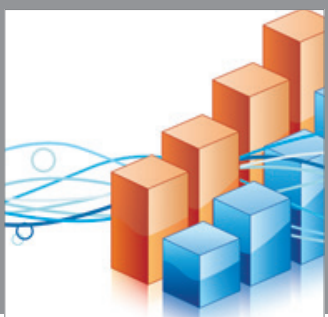

Advances in

Operations Research

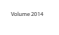

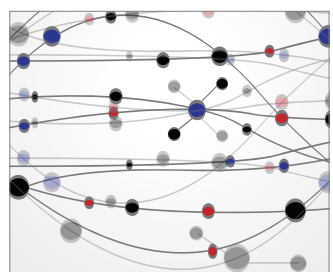

\section{The Scientific} World Journal
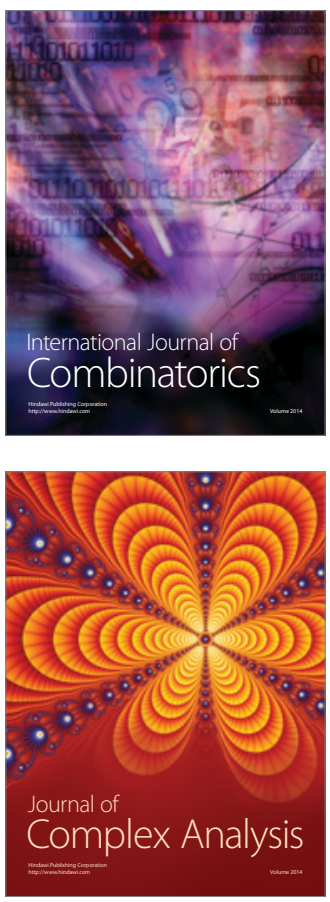

International Journal of

Mathematics and

Mathematical

Sciences
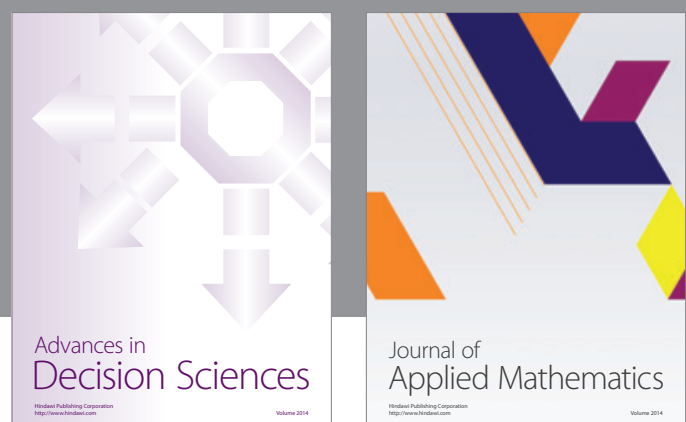

Journal of

Applied Mathematics
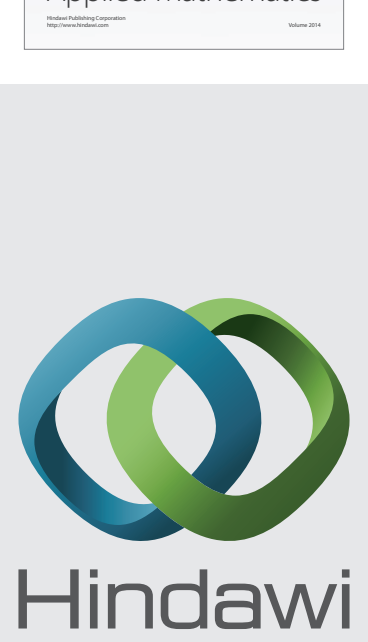

Submit your manuscripts at http://www.hindawi.com
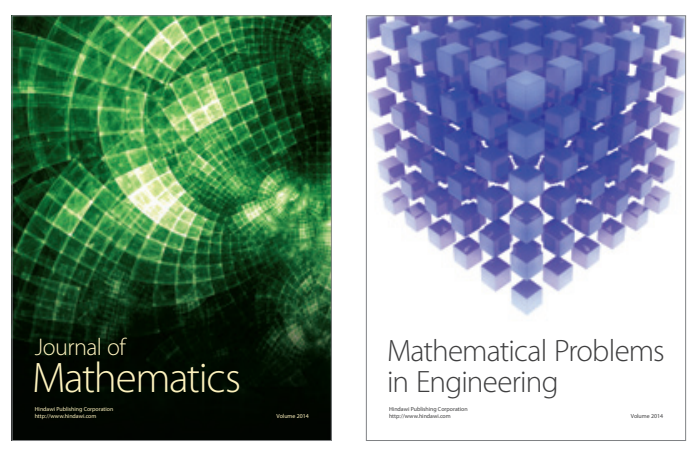

Mathematical Problems in Engineering
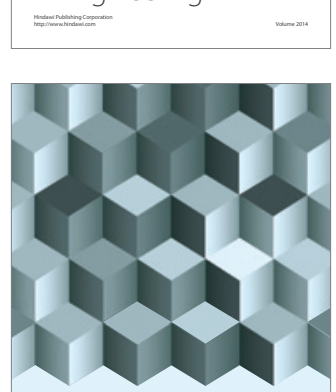

Journal of

Function Spaces
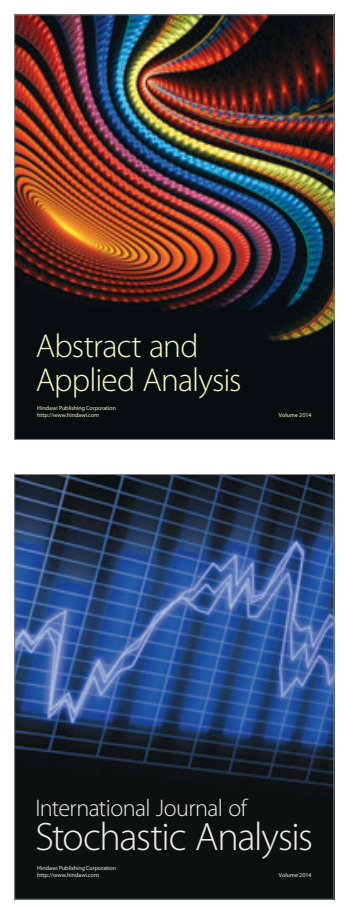

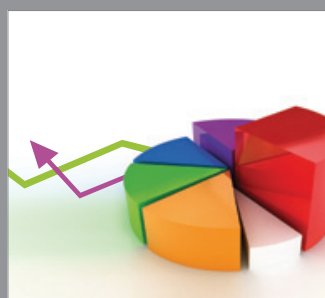

ournal of

Probability and Statistics

Promensencen
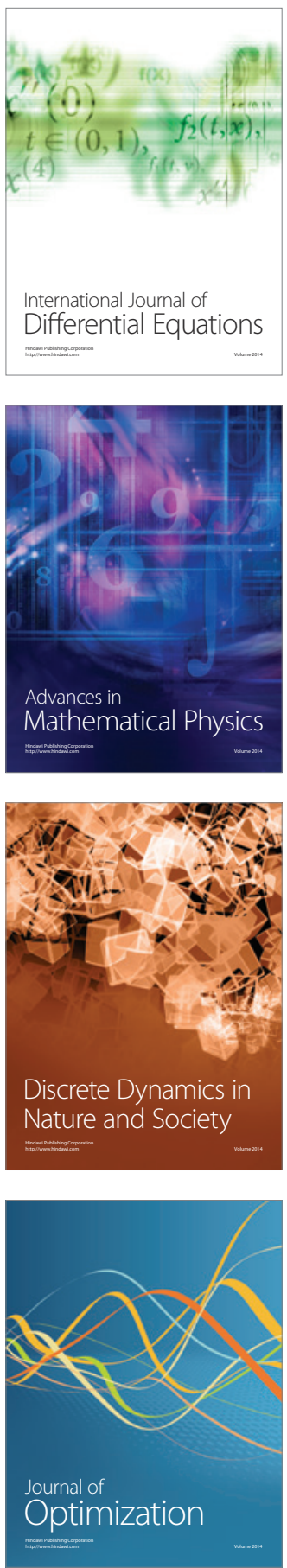